\title{
Experimental characterisation of shear and compaction band mechanisms in porous sandstone by a combination of $A E$ and 3D-DIC
}

\author{
E.-M. Charalampidou ${ }^{1,2, a}$, S.A. Hall ${ }^{2}$, S. Stanchits ${ }^{3}$, G. Viggiani ${ }^{2}$, and H. Lewis ${ }^{1}$ \\ ${ }^{1}$ Heriot-Watt University, Institute of Petroleum Engineering, Edinburgh EH14 4AS, Scotland \\ ${ }^{2}$ Laboratoire 3S-R, CNRS/Grenoble Universities, Domaine Universitaire, BP53, 38041 Grenoble, \\ France \\ ${ }^{3}$ GFZ German Research Centre for Geosciences, Telegrafenberg D423, 14473 Potsdam, Germany
}

\begin{abstract}
Shear and compaction bands represent two fundamental failure modes in sandstones. The identification of these features in both the natural outcrops and the laboratory scale yields useful insights into the mechanisms of localised deformation. Several conventional and advanced experimental full-field methods have been developed in order to shed light into the processes involved, as well as qualify and quantify parameters such as the orientation of these localised bands. In this paper, we present results of two triaxial tests, investigating shear and compaction bands in porous sandstone, using analysis of Acoustic Emission recorded during deformation and 3Dvolumetric Digital Image Correlation based on pre- and post deformation x-ray tomography images.
\end{abstract}

\section{Introduction}

Shear and compaction bands have been both recognised in nature [e.g., 1-3] and reproduced in laboratory [e.g., 4-9] over a number of years. The optimal goal of all such studies is the better understanding of the mechanisms being involved in these failure modes. In fact, compaction localisation in naturally deformed systems can lead to significant porosity and permeability changes. The evolution of features such as shear or compaction bands, can, therefore, have important implication for aquifer management, fluid injection or withdrawal, oil and gas production, storage of $\mathrm{CO}_{2}$ or waste disposal.

\section{Experimental techniques}

At the laboratory scale, the development and propagation of localised deformation in rocks cannot be fully investigated via conventional techniques, i.e., analysis of force and displacement measurements taken at the boundaries of the specimen [10]. Consequently, the use of full-field measurements, (e.g., Ultrasonic Tomography (UT), Acoustic Emission (AE), x-ray Computed Tomography (x-ray CT) or

\footnotetext{
${ }^{a}$ e-mail : elma.charalampidou@pet.hw.ac.uk
} 
Digital Image Correlation (DIC)), has become increasingly common [11]. Such non-conventional laboratory techniques allow measurements of field of properties or processes: e.g., ultrasonic velocity (UT), density (x-rays CT), and displacement/strains (DIC). It is worth noting that each approach has a certain resolution and sensitivity to different physical properties; thus, much can be gained by using them in conjunction. In this work, we combine non-destructive methods such as UT, AE, x-ray CT, 3D-DIC with thin section investigations (destructive method), to study localised deformation in sandstone. In this paper, however, we limit the scope to results obtained by AE analysis and 3D-volumetric DIC on low resolution ( $\sim 90 \mu \mathrm{m}$ voxel size) pre- and post-deformation $\mathrm{x}-$ ray tomography images. Results on high-resolution $(\sim 30 \mu \mathrm{m}$ voxel size $)$ pre- and post-deformation $\mathrm{x}$-ray tomography images are presented elsewhere.

\section{Material and experimental program}

The material studied here is a quartz rich sandstone of $22 \%$ average porosity, coming from the Woustviller quarry in the Vosges Mountains, France [12]. This Vosges Sandstone has a mean grain size diameter of about $300 \mu \mathrm{m}$ and yields $93 \%$ quartz, $5 \%$ feldspar, $1 \%$ clay and $1 \%$ micas [13]. It was shown that the behaviour and failure mode of sandstones are pressure dependent [e.g., 13, 5, 14]. Bésuelle [12] investigated the behaviour of cylindrical specimens of this particular Vosges Sandstone under low (10-30 MPa) and medium (40-60 MPa) confining pressures; at these two pressure ranges, dilating and compacting shear bands developed, respectively. In this work, we validate that compacting shear bands develop in specimens with slightly different geometric configuration loaded under triaxial compression at $50 \mathrm{MPa}$ confining pressure, while, under much higher confining pressures (130-190 MPa) essentially horizontal deformation bands develop. Here, we present two triaxial compression tests, conducted at $50 \mathrm{MPa}$ and $160 \mathrm{MPa}$ confining pressure. Both experiments come from a much larger experimental campaign [15].

Cylindrical specimens (40mm in diameter and $80 \mathrm{~mm}$ in height) were cored perpendicular to the sedimentary bedding. In addition, the specimens had two opposite flattened surfaces throughout their height. This particular geometry is necessary for the UT (not presented in this paper). Bésuelle [12] used cylindrical specimens of the same size and showed that under $50 \mathrm{MPa}$ confining pressure, conjugate compacting shear bands developed in the top part of the samples. To avoid the onset and propagation of conjugate bands, under this pressure range, and to enforce the occurrence of a distinct shear band in the middle of the sample, we machined two notches $(2.5 \mathrm{~mm}$ width and $2.0 \mathrm{~mm}$ high $)$ on the flattened surfaces in different heights (Figure 1). The specimen loaded under $160 \mathrm{MPa}$ did not have any notch (Figure 1).

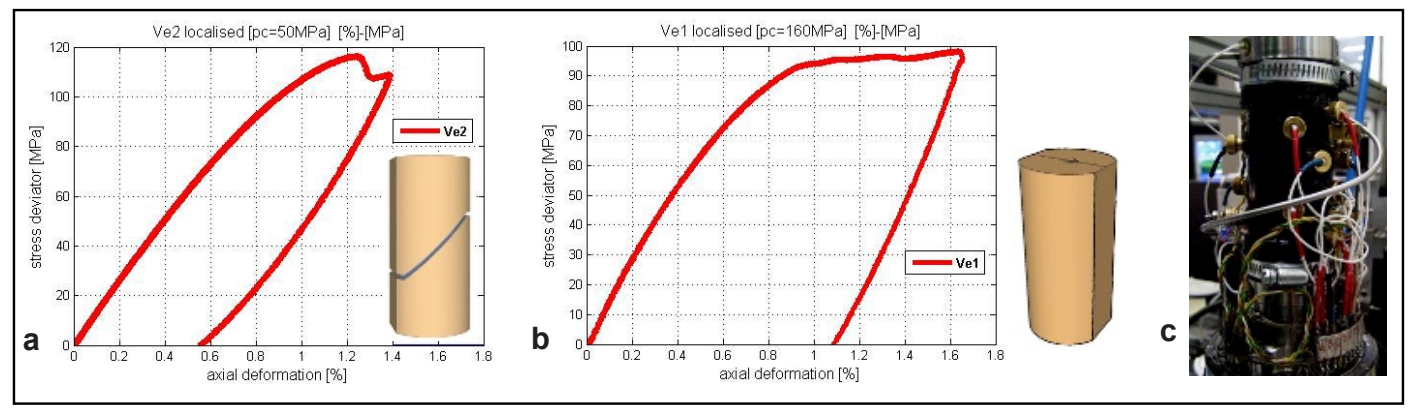

Fig. 1. Stress deviator versus axial strain and schematic picture of the specimens: $a$. shear band, $b$. compaction band, c. Photograph of the AE sensors on the specimen surface.

Both triaxial compression tests (dry) were carried out at GFZ, Potsdam. AE and ultrasonic transmission signals were recorded during the tests using $14 \mathrm{P}$ - and 4S- piezoelectric sensors glued on the surface of the specimens (Figure 1) and 2 P-sensors incorporated in the top and bottom caps. Two vertical and two horizontal strain gauges were installed directly on the rock and an internal load 
transducer was used to monitor applied axial stress. The two specimens were subjected, initially, to hydrostatic loading followed by deviatoric loading. At $50 \mathrm{MPa}$ confining pressure, the axial loading stopped near the peak stress, while, at $160 \mathrm{MPa}$ confining pressure, the axial loading stopped at a higher level of axial strain (compared to that corresponding to the peak stress).

The two experiments (Ve1 and Ve2) presented here, were analysed using AEs recorded during deformation, as well pre- and post- deformation low resolution $(\sim 90 \mu \mathrm{m}$ voxel size) $\mathrm{x}$-ray scans (at Laboratoire 3S-R, Grenoble). Along with the AE analysis (3D), 3D volumetric DIC of the pre- and post- mortem X-ray tomography images will be presented for both specimens.

\section{Results and discussion}

Figure 1 presents the stress deviator $q=\left(\sigma_{a}-\sigma_{r}\right)$, where $\sigma_{a}$ and $\sigma_{r}$ are the axial stress and the confining pressure respectively (the latter being constant during the deviatoric loading), as a function of the axial strain. For Ve2, the loading was stopped at about $1.4 \%$ axial strain and, after complete unloading, $0.54 \%$ axial strain was recovered. In this specimen, shear band developed. For Ve1, the loading was stopped at about $1.65 \%$ axial strain and, after complete unloading, $1.08 \%$ axial strain was recovered. This specimen failed by compaction bands. In the following, results from the AE, 3D volumetric DIC analyses are presented separately for the case of the shear and compaction band.

\subsection{Shear Band}

Figure 2 depicts the time history (in seconds) of the stress deviator and the cumulative AE number, separated into increasing time intervals moving from left to right. Three 2D projections of 3D distributions of AE event locations (one perpendicular and two parallel to the sample axis) are shown in Figures 2b, 2c, 2d. The notches act as stress concentrators; therefore, they encourage localisation to initiate in the regions around them. Indeed, AE events nucleated from the notches in both upper and lower directions, however, they, finally, localised in the region in between the notches (i.e., middle part of the sample).

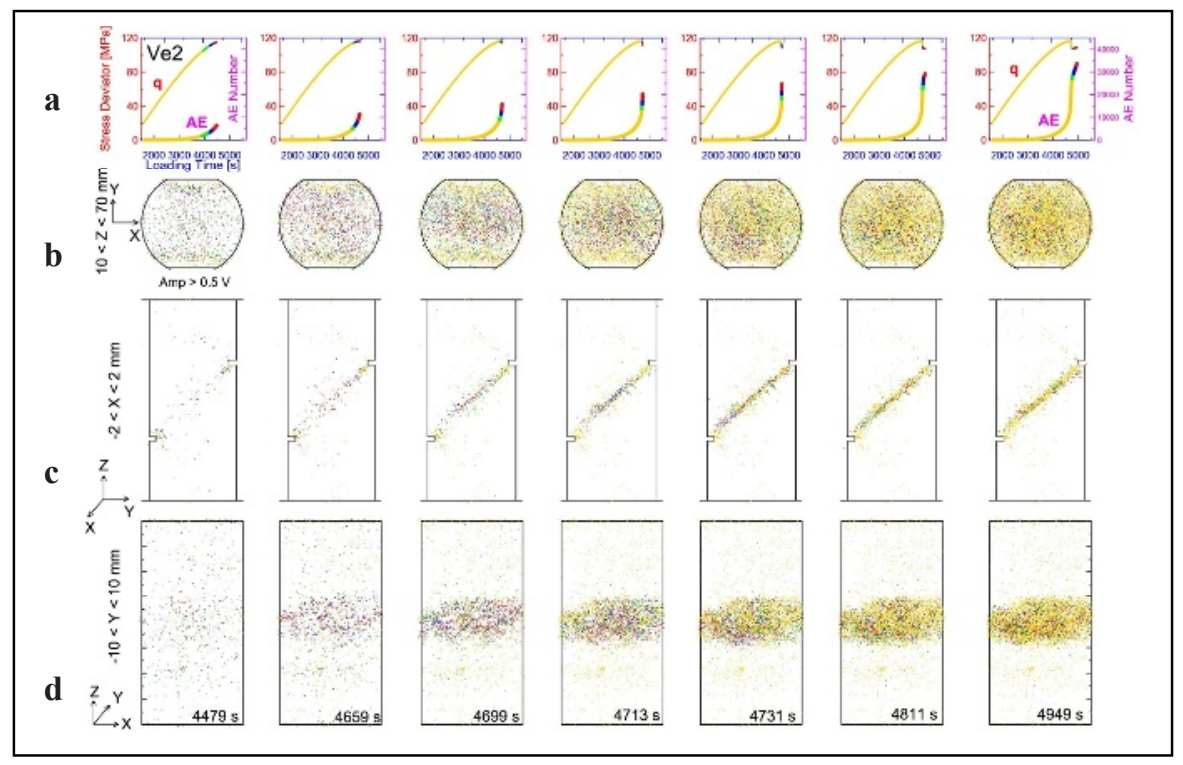

Fig. 2. Stress deviator as a function of the number of $A E$ events in different time intervals; colour changes to highlight most recent events; $b$-d. Maps of AE event locations for the same time intervals viewed perpendicular to the specimen's height, parallel to the specimen's height in a projection perpendicular to the flattened surfaces projections and parallel to the specimen's height in a projection parallel to the flattened surfaces projections. 
Volumetric and shear maximum shear strains were obtained by 3D-volumetric DIC using the code TOMOWARP, based on the work of Hall [16], [see also 17]. The maximum shear strain is $\varepsilon_{s}=\sqrt{2} / 3$ $\left[\left(\varepsilon_{1}-\varepsilon_{2}\right)^{2}+\left(\varepsilon_{2}-\varepsilon_{3}\right)^{2}+\left(\varepsilon_{3}-\varepsilon_{1}\right)^{2}\right]^{1 / 2}$, with $\varepsilon_{1}, \varepsilon_{2}$ and $\varepsilon_{3}$ the major, intermediate and minor principal strains, respectively, while the volumetric strain is $\varepsilon_{v}=\left[\varepsilon_{1}+\varepsilon_{2}+\varepsilon_{3}\right]$. The strain fields, derived from the low resolution $\mathrm{x}$-ray tomography images, indicate that two compacting shear bands initiated from the notches and propagated to the centre of the specimen. Figure 3 presents both shear and volumetric strains in the region where the two bands meet. Strains were calculated over a step equivalent to 3 times the grain size and the calculations were performed in a correlation window equal to 3 times the grain size (Figure 3a). However, it appears that when calculations were performed in a smaller correlation window (equivalent to 1.5 times the grain size) the shear strain field is better resolved, while the volumetric strain field is quite noisy (Figure 3b).

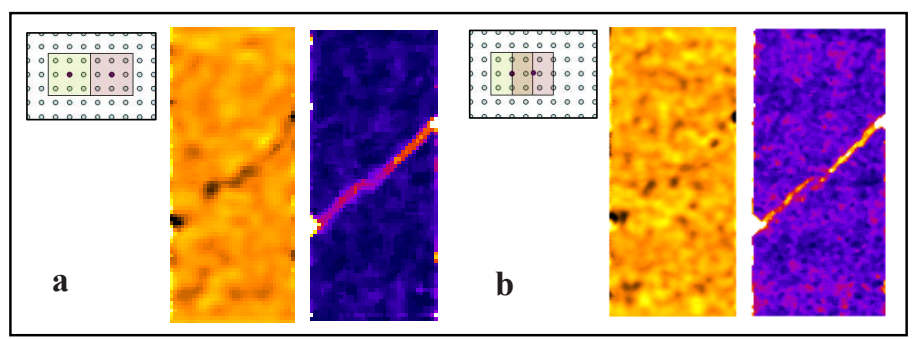

Fig. 3. Volumetric and shear strains in a region where the two compacting shear bands meet. Strains are calculated over a step of 3 grain size and a correlation window equivalent to 3 grain size (a) and 1.5 grain size (b).

The thresholded 3D geometry of the shear band, derived from the shear strain field, is shown in Figure 4a. The vertical column in the central part of the sample is due to ring artefacts. The band appears to be rather planar. Figures $4 \mathrm{~b}$, c show the median values of all vertical projections of volumetric and shear strains, respectively.

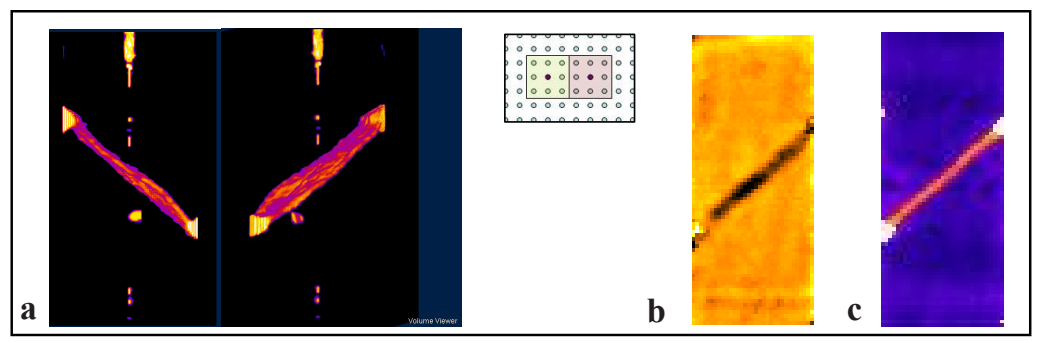

Fig. 4. a. 3D thresholded views of the shear strain field; b. median values of the volumetric strain projections; c. median values of the shear strain projections. All images were calculated for a correlation window equivalent to 3 times the grain size and over a step equivalent to 3 grains

\subsection{Compaction Band}

Similarly to Figure 2, Figure 5 presents the time history (in seconds) of the stress deviator and the cumulative $\mathrm{AE}$ number; the three $2 \mathrm{D}$ projections of $3 \mathrm{D}$ distributions of $\mathrm{AE}$ event locations (one perpendicular and two parallel to the sample axis) are shown in Figures 5b, 5c, 5d. This specimen had no notches. An intensive AE event activity is recorded near the top edge of the sample, while $\mathrm{AE}$ events also localised, near the mid-height of the top part of the sample, in horizontal bands. Once the bands propagated thought the width of the sample, new bands developed. The number of the bands increased with increasing axial strain. Before the unloading, one more band, this time on the 
bottom part of the sample and near the mid-height, developed. These bands are inferred to be compaction bands. It is worth noting that these localised features developed only on the top edge and the middle part of the specimen, while no AE activity was recorded elsewhere. Most likely, that is associated with sample heterogeneity or higher density of bedding planes in those regions.

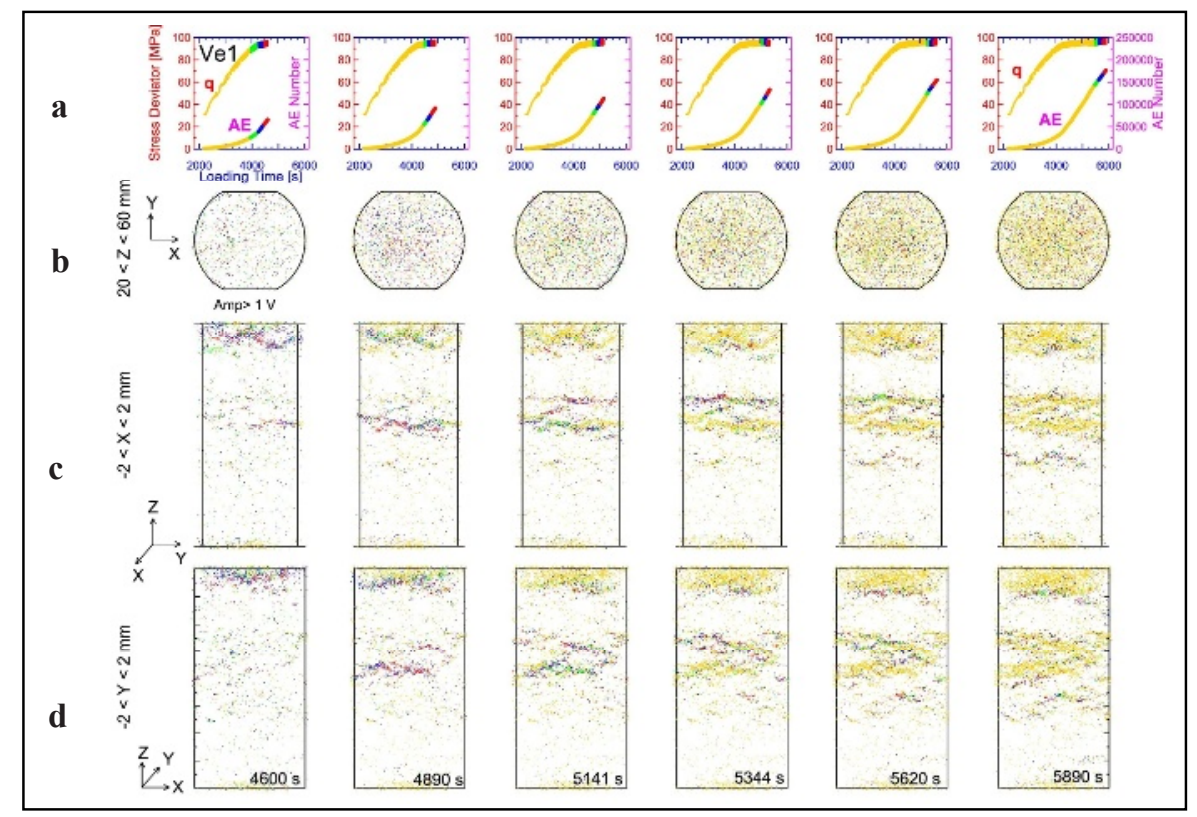

Fig. 5. Stress deviator as a function of the number of $\mathrm{AE}$ events in different time intervals; colour changes to highlight most recent events; b-d. Maps of AE event locations for the same time intervals viewed perpendicular to the specimen's height, parallel to the specimen's height in a projection perpendicular to the flattened surfaces projections and parallel to the specimen's height in a projection parallel to the flattened surfaces projections.

The volumetric and shear strain fields are presented in Figure 6. Strains were calculated over a step equivalent to 1.5 grain size for a correlation window equal to 3 times the grain size. Figure $6 \mathrm{a}$ illustrates the median volumetric and shear field of all vertical projections. A network of compaction bands developed near the top edge and the mid-height of the specimen. Nevertheless, the 3D geometry of the compaction bands appears to be more complex compared to the shear band geometry. Figures $6 \mathrm{~b}$ and $\mathrm{c}$ show two different compaction band networks, inside the volume of the specimen. In both cases, three compaction bands developed above the mid-height of the sample, however, their orientation and propagation change throughout the specimen. Such observation is much better illustrated in Figure 7, which shows the 3D geometry of the compaction bands using the thresholded shear and volumetric strain field.

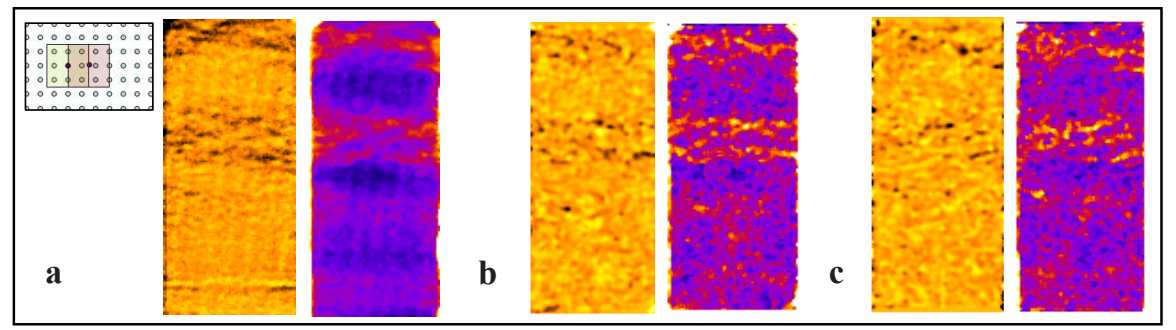

Fig. 6. a. Median values of volumetric and shear strains over all vertical projections; b, c. 2D slices of volumetric and shear strain in different positions inside the specimen. 


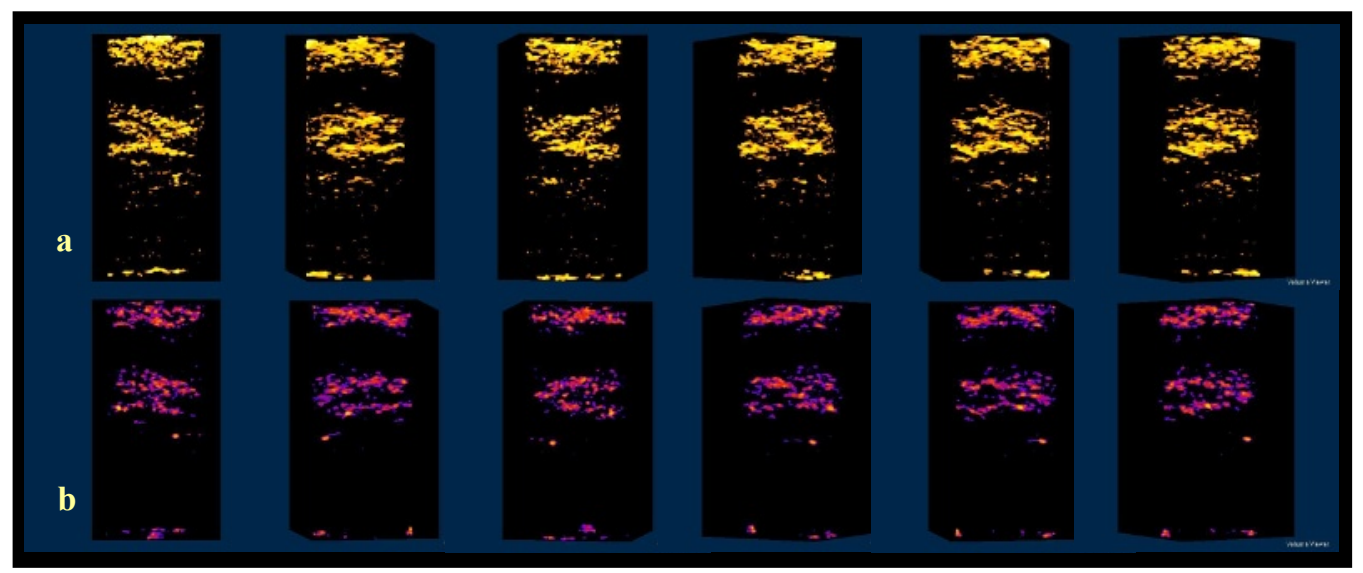

Fig. 7. 2D slices of rotated thresholded 3D view of the compaction bands derived from the volumetric (a) and shear (b) strain fields.

\section{Conclusions}

Results on 3D-volumetric DIC, derived from low resolution (voxel size of $\sim 90 \mu \mathrm{m}$ ) pre- and postdeformation $\mathrm{x}$-ray tomography images, and $\mathrm{AE}$ analysis are presented in this paper to characterise localised deformation in shear and compaction bands. The AE analysis illustrates the onset and evolution of these bands throughout the experiments, while the 3D-volumetric DIC gives a field picture of shear and volumetric strains derived from the deformation process. In both deformation bands compaction and shear have been recorded. The two methods appear to be consistent, since they display similar features in each experiment.

\section{Acknowledgement}

Georg Dresen is gratefully acknowledged for the financial support of the experiments carried out in GFZ. E-M.Charalampidou is supported by an Ali Danesh Scholarship from the Institute of Petroleum Engineering at Heriot-Watt University, Edinburgh.

\section{References}

1. M.A. Antonellini, A. Aydin, D.D. Pollard, J. Struct. Geol., 16, 941-959 (1994)

2. P.N. Mollema, M.A. Antonellini, Tectonophysics, 267, 209-228 (1996)

3. K.R. Sternlof, J.W. Rudnicki and D.D. Pollard, J. Geophys. Res., 110, B11403, doi:10.1029/2005JB003764 (2005).

4. B. Menéndez, W. Zhu, T.-W. Wong, Journal of Structural Geology, Vol.18, No. 1, pp. 1-16 (1996)

5. A. El Bied, J. Sulem, F. Martineau, Int. J. Rock Mech. \& Min. Sci., 39, 917-932 (2002)

6. V. Vajdova and T.-F. Wong, Geophysical Research Letters, VOL. 30, NO. 14, 1775, doi: 10.1029/2003GL017750 (2003)

7. P. Baud, E. Klein, and T.-F. Wong, J. Struct. Geol., 26, 603-624 (2004)

8. J. Fortin, S. Stanchits, G. Dresen, Y. Gueguen, Pure appl. Geophys., 166, 823-841 (2009)

9. S. Stanchits, J. Fortin, Y. Guéguen, G. Dresen, Pure appl. Geophys., 166, 843-868 (2009)

10. J. Desrues, and G. Viggiani, Int. J. Numer. Anal. Meth. Geomech, 18, 179-321, (2004) 
11. G. Viggiani, S. A. Hall, Deformation Characteristics of Geomaterials, IOS Press, Atlanta, USA, p. 3-26 (2008)

12. P. Bésuelle, Phys. Chem. Earth(A), Vol. 26, No. 1-2, pp. 101-106 (2001)

13. P. Bésuelle, J. Desrues, S. Raynaud, Int. J. Rock Mech. \& Min. Sci., 37, 1223-1237 (2000)

14. K. Mair, S. Elphick, I. Main, Geophysical Research Letters, 29, NO. 10, 10.1029/2001GL013964 (2002)

15. E-M. Charalampidou, Experimental study of localised deformation in porous sandstone, $\mathrm{PhD}$ thesis, Heriot-Watt University \& Grenoble University, Edinburgh, Scotland and Grenoble, France, (2010, forthcoming)

16. S.A. Hall, Geophysics, 71, O21-O31 (2006)

17. S.A. Hall, N. Lenoir, G. Viggiani, J. Desrues, and P. Bésuelle, Internl Symp. on Computational Geomechanics, Cote d'Azur, France (2009) 